\title{
Body shape: Implicaciones en el estudio de obesidad y factores asociados
}

\section{Body shape: Implications in the study of obesity and related traits}

Presentación: 6-7/10/2020

\section{Doctorando:}

\section{Jose Pablo Navarro}

Grupo de Investigación en Biología Evolutiva Humana, Instituto Patagónico de Ciencias Sociales y Humanas, Centro Nacional Patagónico, Consejo Nacional de Investigaciones Científicas y Técnicas - Argentina pablo1n7@gmail.com

\section{Director/a:}

\section{Claudio Delrieux}

\section{Co-director/a:}

\section{Rolando González-José}

\section{Resumen}

Basado en el creciente avance de técnicas de computación que permiten describir o predecir características a partir de diferentes representaciones de datos, y tomando como punto de partida la creciente epidemia de obesidad y sobrepeso decretada por la Organización Mundial de la Salud (OMS). Se propone un método de segmentación 3D de escaneos corporales que, junto con técnicas de morfometría geométrica, permite cuantificar la distribución del tejido adiposo en el abdomen. Específicamente, se analizó un conjunto de circunferencias del cuerpo a partir de landmarks posicionados anatómicamente. Posteriormente se calculó un análisis de componentes principales para comparar las variables de forma con los datos antropométricos (peso, altura y circunferencia de cintura y cadera). Los indicadores de forma generados a partir de este nuevo enfoque se correlacionan con el comportamiento de los marcadores clásicos: Índice de Masa Corporal, Índice Cintura Cadera, Índice Cintura Talla.

Palabras clave: Obesidad, Descriptor De Forma Corporal, Landmark.

\begin{abstract}
Based on the growing advancement of computer techniques that allow describing or predicting characteristics from different data representations and taking as a starting point the growing obesity and overweight epidemic decreed by the World Health Organization. A method of 3D segmentation of body scans is proposed that, together with geometric morphometric techniques, allows to quantify the distribution of adipose tissue in the abdomen. Specifically, a set of body circumferences was analyzed, each one defined from anatomically positioned landmarks. Subsequently, a principal component analysis was calculated to compare the shape variables with the anthropometric data (weight, height and waist and hip circumference). The shape indicators generated from this new approach are correlated with the behavior of the classic markers: Body Mass Index, Waist-Hip Index, Waist-Height Index.
\end{abstract}




\section{Introducción}

En la actualidad, el diagnóstico y tratamiento de obesidad están basados en indicadores clásicos como la relación entre peso y altura (Índice de Masa Corporal o IMC) (Keys, 1972), la circunferencia de cintura y cadera (Índice Cintura Cadera o ICC) o el Índice de Cintura Talla (ICT) (Ashwell, 2012; Larsson, 1984; Lapidus 1984). En algunos casos, se utilizan evaluaciones antropométricas más complejas y costosas para obtener un marco general de proporciones corporales y distribución de grasa. Establecer un umbral adecuado o puntos de corte en los índices para predecir estados nutricionales (como obesidad y sobrepeso), está directamente relacionado con las características de la población de referencia. Estudios anteriores indican las diferencias observadas entre las poblaciones de las islas de Asia, Europa o el Pacífico sobre los estándares de IMC para clasificar el sobrepeso y la obesidad en adultos (Carroll, 2008; Gu, 2018; Kagawa, 2007; Madan, 2012; Rønn, 2017). Estas diferencias también son reportadas para los índices ICC e ICT. Este problema sigue sin estar claro en poblaciones mixtas o mestizas, como en grandes partes de América Latina. Debido a su extensa mezcla entre las tres principales poblaciones continentales (africanos, europeos y nativos americanos), los latinoamericanos tienen un fondo biológico complejo (Koehl y Long, 2018; Norris, 2018; Ruiz-Linares, 2014).

En un estudio reciente se evaluó mediante regresión lineal múltiple, la relación entre obesidad definida como IMC, ICT y ICC, ascendencia genómica y estado socioeconómico en una muestra de latinoamericanos mezclados (6776 voluntarios adultos de Brasil, Chile, Colombia, México y Perú). La proporción de obesidad depende en gran medida de la población e índice considerado, los cuales no se superponen constantemente al clasificar la obesidad (Ruderman, 2019). Es importante establecer un corte específico para el gran porcentaje de la población mestiza de América Latina y estudiar cuáles serían los parámetros para un diagnóstico correcto de sobrepeso y obesidad. Una mejor respuesta a este problema podría incluir el registro y el análisis de imágenes en 3D como se propone en este trabajo en el cual se combinan técnicas de computación aplicadas a la antropología.

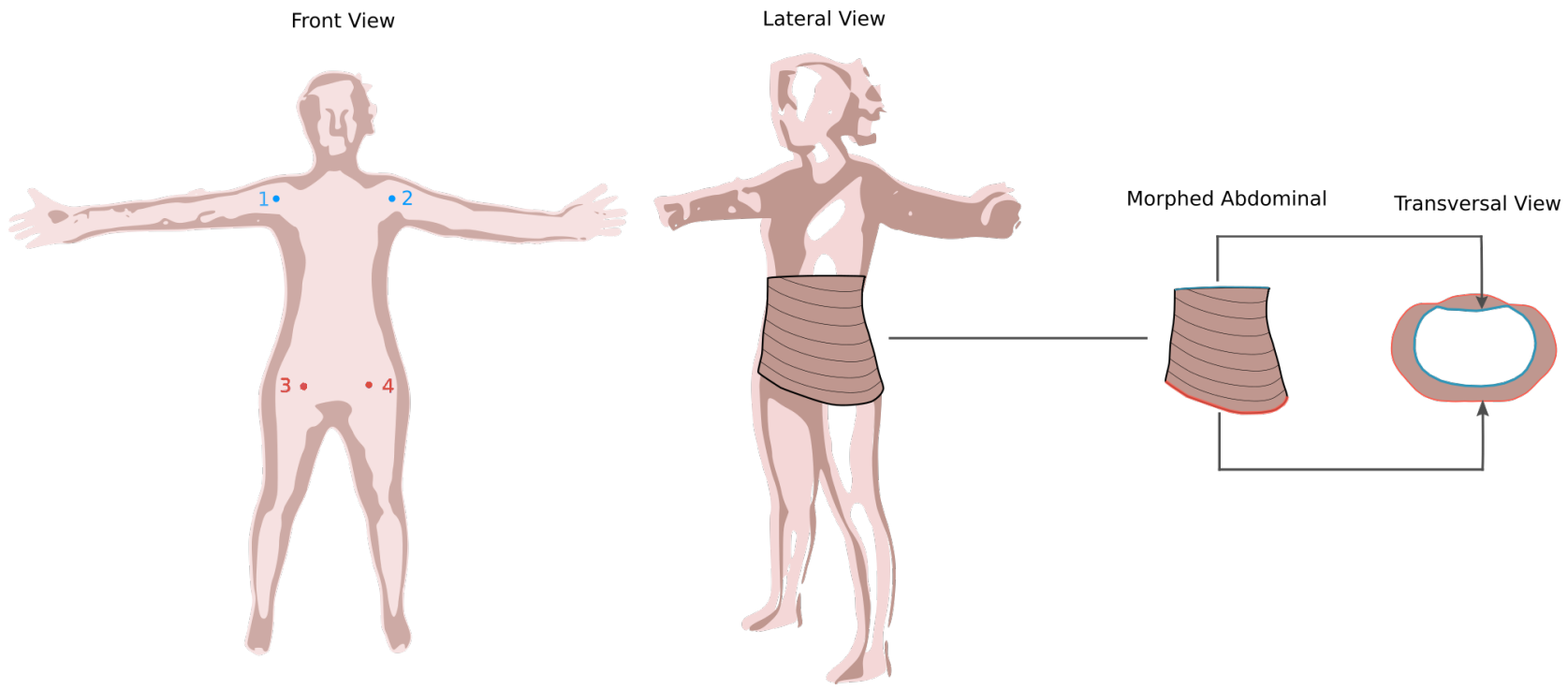

Figura 1: Ejemplo de imagen 3D del conjunto de datos, junto con la configuración de landmarks usada para la extracción de las características de la forma corporal. Los landmarks $1-2$ corresponde a la articulación del hombro (glenohumeral) y los landmarks $3-$ 4 a los punto donde articula la cabeza del fémur en la cadera (Navarro, 2019).

\section{Desarrollo}

Para este procedimiento se utilizaron los datos colectados bajo el protocolo del Proyecto 3DLab. Los mismos fueron obtenidos durante una convocatoria a personas mayores de 18 años en la ciudad de Puerto Madryn. La campaña de recolección de datos consistió en la toma de medidas antropométricas clásicas e índices asociados al estado nutricional de los voluntarios acompañado 
por la toma de imágenes 3D por medio de un dispositivo de escaneo. El grupo de voluntarios contó con 154 individuos en total con un promedio de edad de 39 años. El escáner utilizado fue Structure Sensor (desarrollado por Occipital Inc., San Francisco, California, el cual fue fabricado como un accesorio de uso general para dispositivos iPad (4ta generación, Apple Inc., Cupertino, California).

Con el objetivo de no sesgar negativamente el experimento, se descartaron los individuos de la muestra que contenían deformaciones anormales ubicadas en el torso de sus representaciones 3D. Estas anomalías se atribuyen a errores de escaneo, producidos por el dispositivo al capturar algún tipo superficie o simplemente por error del operador. Considerando la muestra ( $\mathrm{n}=112$ ), 15,2 \% de la muestra fueron considerados obesos de acuerdo a su IMC, 31,2 \% con sobrepeso, y 53,6 \% saludables. El porcentaje de voluntarios con obesidad según el criterio de corte para ICT es de $81,2 \%$. La misma tendencia se muestra de acuerdo al ICC con $60 \%$ de hombres y $69 \%$ de mujeres obesas.

En las imágenes 3D fueron colocados cuatro puntos de anclaje anatómico también denominados landmarks. Estos landmarks fueron ubicados por un operario capacitado ya que se requiere tener experiencia y conocimientos de anatomía. La utilización de estos landmarks permite obtener puntos de correspondencia entre todos los modelos de la muestra. Es decir, cómo estos puntos están posicionados anatómicamente es posible ubicarlos en cualquier representación del cuerpo humano. En la figura 1 se puede observar la posición de estos cuatro puntos de anclaje situados sobre un modelo ejemplo. Los landmarks $1-2$ corresponde a la articulación del hombro (glenohumeral) y los landmarks 3-4 a los punto donde articula la cabeza del fémur en la cadera.

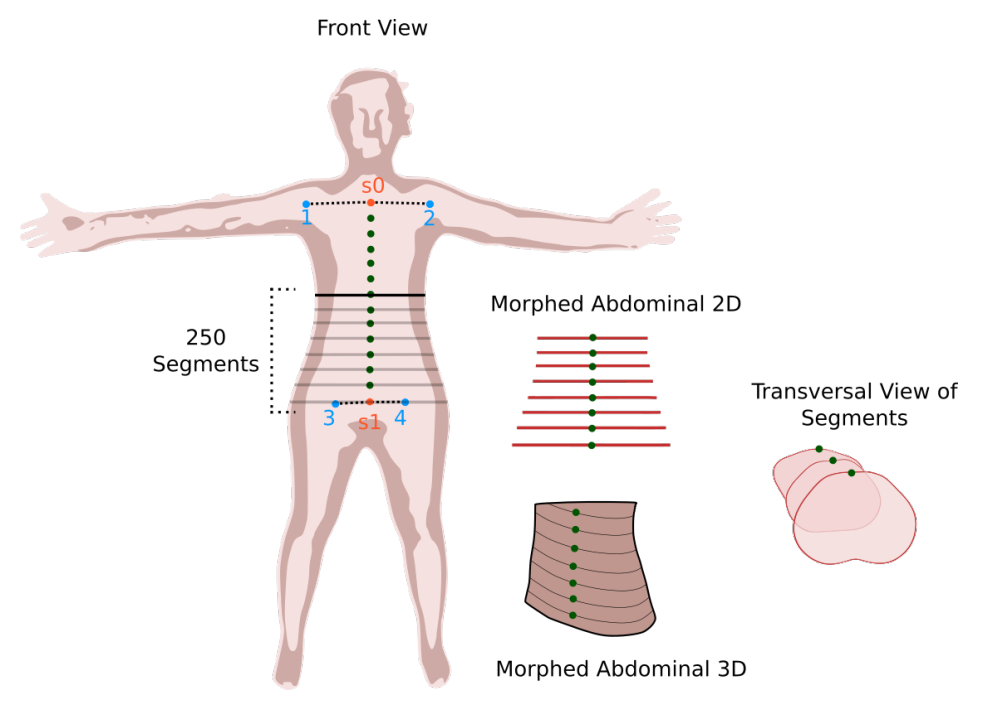

Figura 2: Localización de los semi-landmarks s0 y s1 en naranja. Los 500 puntos ajustados por el criterio de mínimo Bending Energy en verde. El área abdominal descripta en distintas vistas (2D, 3D y vista transversal.) generados con 250 segmentos (Navarro, 2019).

El escáner 3D es una herramienta potente a la hora de capturar muestras a gran escala y necesaria para realizar estudios de población (Jaeschke, 2015; Löffler-Wirth, 2016). El problema con estas representaciones es como extraer características (features) discriminantes que permitan estudiar los objetos capturados de una manera eficiente. Para resolver este problema, el presente procedimiento propone utilizar los landmarks, anteriormente nombrados, como referencia a la hora de extraer features. Es por esto que se calcularon dos puntos s0 y s1 entre los pares de landmarks 1,2 y 3,4. Como estos puntos fueron obtenidos a partir de landmarks se los puede denominar semi-landmarks (Ver Figura 2). A partir de acá, se calculó la distancia Euclidiana entre los semi-landmarks, con el fin de obtener información sobre la posición relativa al área abdominal. Utilizando la distancia que se calculó previamente se posicionaron 498 puntos a lo largo del área correspondiente entre s0 y s1 y se ajustaron con el criterio de menor Bending Energy (Gunz y Mitteroecker, 2013). Finalizado este proceso se obtuvieron 500 semi-landmarks (incluyendo s0 y s1) posicionados sobre el modelo 3D (Ver Figura 2). La zona inferior del área abdominal constituye la región en donde se centra 
la mayor concentración de grasa corporal, por lo cual este es un área de interés para el estudio de obesidad y sobrepeso. Con esto en mente, se utilizó los 250 semi-landmarks inferiores que, al estar fijados a landmarks anatómicos, constituyen exactamente la mitad inferior.

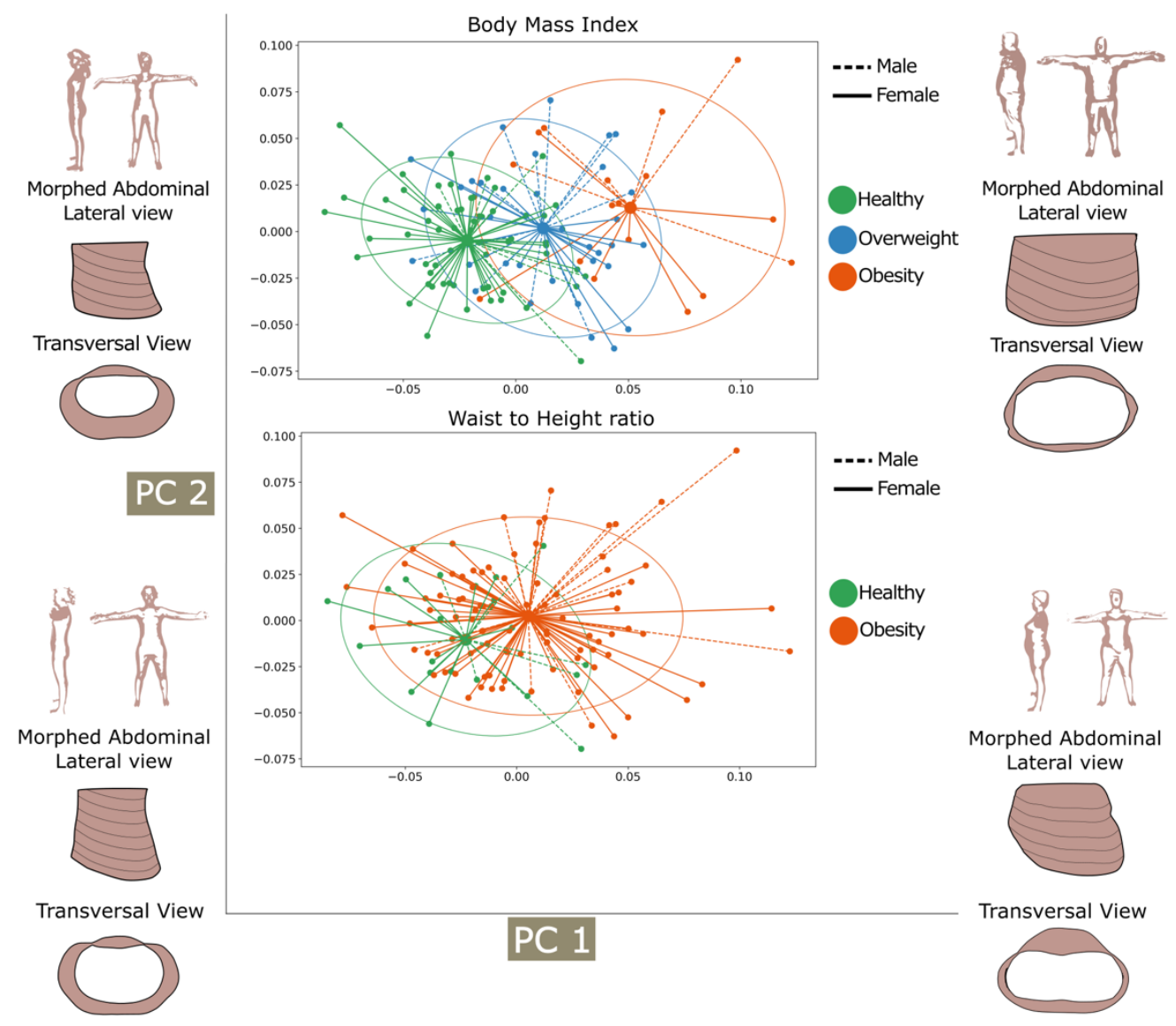

Figura 3: Análisis de componentes principales. Gráfico de dispersión y variación de forma y distribución de la grasa abdominal con el descriptor propuesto. Elipses coloreadas basadas en IMC, ICT y sus estados nutricionales. Los cambios de forma representan el cambio de forma promedio observado en los extremos de los ejes (Navarro, 2019).

A partir de aquí, y utilizando operaciones básicas en 3D, se generaron 250 láminas de corte, es decir 250 prismas rectangulares en tres dimensiones (con 0,01 espesor). Estos rectángulos fueron alineados con el modelo (uno con cada semi-landmarks) y, mediante operaciones de intersección, se obtuvieron 250 segmentos del área inferior abdominal. Estos segmentos son utilizados como dato principal para construir un espacio de feature capaz de detectar y analizar las diferencias de forma entre sexos y estado nutricional. Las 250 circunferencias de cada voluntario fueron proyectadas dentro de un espacio computado a través de Análisis de Componentes Principales (PCA). El primer componente principal (PC1) y el segundo componente principal (PC2) explican $12,5 \%$ y 7,5 \% de la variación respectivamente. En Figura 3 se observa la distribución de los individuos dentro del espacio definido por estos dos primeros componentes. Además se visualiza la variación de la forma abdominal a lo largo de los ejes.

Recorriendo el primer eje (PC1) desde los valores negativos hacia los positivos, los valores discriminan casi por completo los cambios anteriores-posteriores en los torso basado en reconstrucciones $3 \mathrm{D}$ correspondientes a los voluntarios con menor adiposidad central a aquellos con sobrepeso marcado. En el segundo eje, todos los valores negativos corresponden con torsos clasificados como saludables (de acuerdo a los tres índices). Estas variaciones de forma están afectadas por el efecto combinado del estado nutricional y el dimorfismo sexual de los individuos. 
La variación en la forma que describe la obesidad abdominal se expuso a lo largo de PC1 y PC2 (Figura 3) donde el estado nutricional de los individuos están indicados con diferentes colores. Las elipses agrupan a los voluntarios de acuerdo con los valores de corte referidos para cada índice como "Saludable / Sobrepeso / Obesidad" para el IMC o "Saludable / Obesidad" para ICT. Las líneas coloreadas indican la distancia de cada voluntario al centroide de la distribución. Puede observarse visualmente que cada persona puede clasificarse en más de una categoría, de acuerdo con diferentes índices. Esta discrepancia es especialmente notable entre IMC y ICT. La clasificación es congruente solo para aquellos fenotipos considerados como obesos en relación con

su peso y estatura, pero varía notablemente para el resto. En el espacio definido por los dos ejes las diferentes elipses se superponen, y muchos de estos voluntarios están más cerca del centroide de una categoría cercana que el que corresponde según su designación siguiendo cada uno de los tres índices.

La distribución del tejido adiposo abdominal debe considerarse como un aspecto de la forma y no como una relación lineal entre las medidas antropométricas. Se plantea la cuestión de si el análisis de varianza de un segmento de reconstrucción 3D puede verse como un enfoque más preciso para el diagnóstico de sobrepeso y obesidad. Para evaluar esta hipótesis, se realizaron análisis de regresión lineal entre el primer y el segundo eje (PCA calculado para hombres y mujeres por separado) y los tres valores de índice clásicos: IMC, ICT y ICC. Las regresiones fueron estadísticamente significativas para PC1.

\section{Conclusiones}

El enfoque clásico utilizado en todo el mundo para definir el sobrepeso y la obesidad es el IMC, la cual es una herramienta económica y rápida de usar durante el diagnóstico clínico. Sin embargo, el IMC y otros índices relacionados tienden a no describir la obesidad con precisión, debido a las variaciones dentro y entre la población en la distribución de grasa abdominal (Chandalia, 2007; Gallagher, 2000; Wulan, 2010). El método presentado aquí permite a los investigadores obtener de manera masiva información sobre la forma y variabilidad del cuerpo humano. Además, este tipo de enfoque provee metodologías apropiadas para detectar la obesidad central (Medina- Inojosa, 2016) y determinar el volumen y la superficie del cuerpo junto con medidas antropométricas con gran precisión (Kuehnapfel, 2016, 2017; Liu, 2017). Las imágenes 3D y los dispositivos para obtenerlas se han vuelto cada vez más accesibles y se deben desarrollar nuevos métodos de análisis que faciliten el procesamiento de esta información. En nuestra vida diaria, ya se utilizan tecnologías con gran capacidad de cómputo, como teléfonos inteligentes, tabletas y computadoras personales, con un potencial para la práctica profesional. Los avances en los métodos de análisis 3D permitirán a los médicos aprovechar las nuevas tecnologías (por ejemplo, aplicaciones para teléfonos inteligentes) para obtener definiciones más realistas de la forma del cuerpo en diferentes condiciones de salud y sus causas relacionadas.

\section{Referencias}

Keys, A., FLAMINIO, F., KARVONEN, M., \& KI-MURA, N. (1972). HL TAYLOR. Indices of relative weight and obesity. J. Chronic Diseases, 25, 329.

Ashwell, M., Gunn, P., \& Gibson, S. (2012). Waist-to-height ratio is a better screening tool than waist circumference and BMI for adult cardiometabolic risk factors: systematic review and meta-analysis. Obesity reviews, 13(3), 275-286.

Larsson, B. K. L. L. P. G., Svärdsudd, K., Welin, L., Wilhelmsen, L., Björntorp, P., \& Tibblin, G. D. (1984). Abdominal adipose tissue distribution, obesity, and risk of cardiovascular disease and death: 13 year follow up of participants in the study of men born in 1913. $\mathrm{Br}$ Med J (Clin Res Ed), 288(6428), 1401-1404.

Lapidus, L., Bengtsson, C., Larsson, B. O., Pennert, K., Rybo, E., \& Sjöström, L. (1984). Distribution of adipose tissue and risk of cardiovascular disease and death: a 12 year follow up of participants in the population study of women in Gothenburg, Sweden. $\mathrm{Br} M e d$ $J$ (Clin Res Ed), 289(6454), 1257-1261.

Carroll, J. F., Chiapa, A. L., Rodriquez, M., Phelps, D. R., Cardarelli, K. M., Vishwanatha, J. K., ... \& Cardarelli, R. (2008). Visceral fat, waist circumference, and BMI: impact of race/ethnicity. Obesity, 16(3), 600-607.

Gu, Z., Zhu, P., Wang, Q., He, H., Xu, J., Zhang, L., ... \& Zhang, L. (2018). Obesity and lipid-related parameters for predicting metabolic syndrome in Chinese elderly population. Lipids in Health and Disease, 17(1), 289. 
Kagawa, M., Binns, C. B., \& Hills, A. P. (2007). Body composition and anthropometry in Japanese and Australian Caucasian males and Japanese females. Asia Pac J Clin Nutr, 16(Suppl 1), 31-36.

Madan, A., Archambeau, O. G., Milsom, V. A., Goldman, R. L., Borckardt, J. J., Grubaugh, A. L., ... \& Frueh, B. C. (2012). More than black and white: differences in predictors of obesity among Native Hawaiian/Pacific Islanders and European Americans. Obesity, 20(6), $1325-1328$.

Rønn, P. F., Andersen, G. S., Lauritzen, T., Christensen, D. L., Aadahl, M., Carstensen, B., \& Jørgensen, M. E. (2017). Ethnic differences in anthropometric measures and abdominal fat distribution: a cross-sectional pooled study in Inuit, Africans and Europeans. $J$ Epidemiol Community Health, 71(6), 536-543.

Koehl, A. J., \& Long, J. C. (2018). The contributions of admixture and genetic drift to diversity among post-contact populations in the Americas. American journal of physical anthropology, 165(2), 256-268.

Norris, E. T., Wang, L., Conley, A. B., Rishishwar, L., Mariño-Ramírez, L., Valderrama-Aguirre, A., \& Jordan, I. K. (2018). Genetic ancestry, admixture and health determinants in Latin America. BMC genomics, 19(8), 75-87.

Ruiz-Linares, A., Adhikari, K., Acuña-Alonzo, V., Quinto-Sanchez, M., Jaramillo, C., Arias, W., ... \& Gómez-Valdés, J. (2014). Admixture in Latin America: geographic structure, phenotypic diversity and self-perception of ancestry based on 7,342 individuals. PLoS Genet, 10(9), e1004572.

Ruderman, A., Pérez, L. O., Adhikari, K., Navarro, P., Ramallo, V., Gallo, C., ... \& Canizales-Quinteros, S. (2019). Obesity, genomic ancestry, and socioeconomic variables in Latin American mestizos. American Journal of Human Biology, 31(5), e23278.

Navarro, P., Ramallo, V., Cintas, C., Ruderman, A., de Azevedo, S., Paschetta, C., ... \& González-José, R. (2020). Body shape: Implications in the study of obesity and related traits. American Journal of Human Biology, 32(2), e23323.

Jaeschke, L., Steinbrecher, A., \& Pischon, T. (2015). Measurement of waist and hip circumference with a body surface scanner: feasibility, validity, reliability, and correlations with markers of the metabolic syndrome. PloS one, 10(3), e0119430.

Löffler-Wirth, H., Willscher, E., Ahnert, P., Wirkner, K., Engel, C., Loeffler, M., \& Binder, H. (2016). Novel anthropometry based on 3D-bodyscans applied to a large population-based cohort. PloS one, 11(7), e0159887.

Gunz, P., \& Mitteroecker, P. (2013). Semilandmarks: a method for quantifying curves and surfaces. Hystrix, the Italian journal of mammalogy, 24(1), 103-109.

Chandalia, M., Lin, P., Seenivasan, T., Livingston, E. H., Snell, P. G., Grundy, S. M., \& Abate, N. (2007). Insulin resistance and body fat distribution in South Asian men compared to Caucasian men. PloS one, 2(8), e812.

Gallagher, D., Heymsfield, S. B., Heo, M., Jebb, S. A., Murgatroyd, P. R., \& Sakamoto, Y. (2000). Healthy percentage body fat ranges: an approach for developing guidelines based on body mass index. The American journal of clinical nutrition, 72(3), 694-701.

Wulan, S. N., Westerterp, K. R., \& Plasqui, G. (2010). Ethnic differences in body composition and the associated metabolic profile: a comparative study between Asians and Caucasians. Maturitas, 65(4), 315-319.

Medina-Inojosa, J., Somers, V. K., Ngwa, T., Hinshaw, L., \& Lopez-Jimenez, F. (2016). Reliability of a 3D body scanner for anthropometric measurements of central obesity. Obesity, open access, 2(3).

Kuehnapfel, A., Ahnert, P., Loeffler, M., \& Scholz, M. (2017). Body surface assessment with 3D laser-based anthropometry: reliability, validation, and improvement of empirical surface formulae. European journal of applied physiology, 117(2), 371-380.

Kuehnapfel, A., Ahnert, P., Loeffler, M., Broda, A., \& Scholz, M. (2016). Reliability of 3D laser-based anthropometry and comparison with classical anthropometry. Scientific Reports, 6(1), 1-11.

Liu, X., Niu, J., Ran, L., \& Liu, T. (2017). Estimation of human body volume (BV) from anthropometric measurements based on threedimensional (3D) scan technique. Aesthetic Plastic Surgery, 41(4), 971-978. 